\title{
Deregulation of the cell polarity protein Lethal giant larvae 2 (Lgl2) correlates with gastric cancer progression
}

\author{
Kyung Han Nam • Min A. Kim • Gheeyoung Choe • \\ Woo Ho Kim • Hye Seung Lee
}

Received: 17 August 2013/Accepted: 24 November 2013/Published online: 13 December 2013

(C) The International Gastric Cancer Association and The Japanese Gastric Cancer Association 2013

\begin{abstract}
Background We investigated the roles of Lethal giant larvae 2 ( $\mathrm{Lgl} 2)$, an epithelial cell polarity protein, during gastric carcinogenesis and gastric cancer (GC) progression and evaluated the correlation of Lgl2 with epithelial-mesenchymal transition (EMT) markers.

Methods Lgl2 protein and mRNA expression were determined by immunohistochemistry and mRNA in situ hybridization in a large series of GC and preneoplastic lesions. Additionally, expression of 7 EMT markers was examined by immunohistochemistry.

Results Loss of membrane Lgl2 staining in GC was observed in 347 of 409 GCs. Lgl2 loss was associated with diffuse histological type $(P<0.001)$, advanced stage $(P=0.021)$, and worse prognosis $(P=0.047)$. Furthermore, Lgl2 loss correlated with reduced E-cadherin expression $(P<0.01)$ and increased expression of vimentin $(P<0.01)$. Combined analysis of Lgl2 and the EMT markers, S100A4 and MMP2, improved predictions of patient outcomes. During gastric carcinogenesis,
\end{abstract}

Electronic supplementary material The online version of this article (doi:10.1007/s10120-013-0324-0) contains supplementary material, which is available to authorized users.

K. H. Nam · G. Choe · H. S. Lee $(\square)$

Department of Pathology, Seoul National University Bundang

Hospital, 173-82 Gumi-ro, Bundang-gu, Seongnam-si,

Gyeonggi-do 463-707, Korea

e-mail: hye2@snu.ac.kr

K. H. Nam - M. A. Kim - G. Choe - W. H. Kim

Department of Pathology, Seoul National University

College of Medicine, Seoul, Korea

W. H. Kim

Cancer Research Institute, Seoul National University

College of Medicine, Seoul, Korea membrane expression of $\mathrm{Lgl} 2$ was progressively lost in $4 \%$ of normal mucosa, $75 \%$ of intestinal metaplasia, $58 \%$ of gastric dysplasia, $69 \%$ of intestinal type GC, and $96 \%$ of diffuse type GC.

Conclusions Our results suggest that Lg12 loss occurs at an early stage of gastric carcinogenesis and contributes to GC progression.

Keywords Stomach neoplasm - Lg12 . Immunohistochemistry · mRNA in situ hybridization . Epithelial-mesenchymal transition

\section{Introduction}

Although the incidence of gastric cancer has decreased in recent decades, it is the fourth most common cancer and the second leading cause of cancer-related deaths worldwide [1]. Despite considerable advances in cancer therapy, prognosis of patients with gastric cancer remains poor due to the advanced stage of the disease at the time of diagnosis, and limited availability of curative treatment at these stages [1]. Thus, gastric cancer is a major public health issue. Since multiple genetic alterations in tumor suppressor genes, oncogenes, cell adhesion molecules, growth factors/receptor systems, and DNA repair genes are involved in gastric carcinogenesis, the identification of mechanisms underlying this malignancy is important [2]. A better understanding of the biology of gastric cancer may contribute to identifying novel diagnostic and prognostic markers, as well as new therapeutic strategies.

Cell polarity is a crucial phenomenon in many biological processes and is required for normal tissue integrity and tissue homeostasis [3, 4]. Accumulating evidence has revealed that defects in cell polarity may result in tissue 
disorganization and subsequently lead to the initiation and progression of cancer. Therefore, loss of cell polarity is considered one of the hallmarks of tumorigenicity, as well as a precondition for human epithelial cancer [5]. Lethal giant larvae ( $\operatorname{lgl}$ ), first discovered in Drosophila as a tumor suppressor, is an essential component of epithelial apical-basal polarity [5-7]. The human homologs of $\lg l, \mathrm{Lgl1}$ and $\mathrm{Lgl} 2$ (also known as Hugl-1 and Hugl-2), localize to the basolateral domain in epithelial cells, and have conserved functions in the establishment and maintenance of cell organization [5, 7]. A growing body of evidence suggests that loss of $\mathrm{Lgl}$ function results in the disruption of polarized epithelial organization, as well as affecting signaling pathways that regulate cell growth, which are linked to human cancers [4, $7,8]$. Reduced expression of Lgl1 has been found during the progression of a variety of human epithelial malignancies, including malignant melanoma, colorectal, ovarian, breast, lung, prostate, endometrial, and hepatocellular carcinoma [5, 9-13]. Additionally, loss of Lgl1 has been correlated with advanced tumor stage, lymph node metastasis and poor prognosis $[5,10,11,13]$. Down-regulation of $\mathrm{Lgl} 2$ has been observed in breast and colorectal cancer and has been associated with cancer progression [14-16]. Lgl2 promoter activity has been reported to be repressed by Snail1 and ZEB1, the key transcription factors associated with epithelial-mesenchymal transition (EMT), in colorectal and breast cancer cell lines, which led to the disorganization of epithelial architecture, suggesting an interaction between Lgl2 and EMT-related proteins [14, 16]. In the stomach, Lgl2 expression is maintained in all reactive and chronic inactive gastritis, but it is lost or aberrantly localized in a high proportion of adenocarcinomas and dysplasias including Barrett gastric foveolar dysplasia [17-19]. Thus, Lgl2 has been proposed to be a potential marker for diagnosing dysplasia and adenocarcinoma. However, the diagnostic role of $\mathrm{Lgl} 2$ has not been validated in a large scaled cohort, and the clinicopathologic relevance of $\mathrm{Lgl} 2$ expression in gastric cancer, specifically the correlation with EMT-related proteins, has not been studied in detail.

The present study evaluated the clinical significance of Lg12 expression in gastric cancer and examined the correlation of Lg12 expression with the expression of EMTrelated proteins. Based on previous studies, we also aimed to validate the diagnostic role of $\mathrm{Lgl} 2$ expression in differentiating gastric dysplasia and cancer from non-neoplastic gastric mucosa, and we examined the role of $\mathrm{Lgl} 2$ in gastric carcinogenesis. To distinguish between membrane and cytoplasmic expression of $\mathrm{Lgl}$, we performed immunohistochemistry of $\mathrm{Lgl} 2$ in a large series of gastric cancer tissue samples from surgically resected and endoscopically removed specimens. Additionally, we performed mRNA in situ hybridization of $\mathrm{Lgl} 2$ and analyzed the correlation between mRNA transcription and protein expression.

\section{Patients and methods}

\section{Patients}

Surgically resected gastric cancer tissue specimens were obtained from 409 patients who underwent a gastrectomy at the Department of Pathology, Seoul National University College of Medicine between January and December, 2004. The age, sex, lymphatic invasion, and pTNM stage (according to the American joint committee on cancer, seventh edition) [20] were evaluated by reviewing medical charts and pathological records. The median age of the patients was 59.0 years (range 24-86) and $88.5 \%$ of the patients had undergone curative resection (R0 according to the international union against cancer guidelines). The series included 300 advanced and 109 early gastric cancers. According to the 7th edition of pTNM staging, there were 137 cases of stage I, 91 of stage II, 128 of stage III, and 53 of stage IV gastric cancer. No patient had received preoperative chemotherapy or radiotherapy. Glass slides were reviewed to determine the histological type (according to WHO and Lauren's classifications) [21, 22]. The series included 183 intestinal types and 226 diffuse types of gastric cancer. The clinical outcome of the patients was followed from the date of surgery to either the date of death or December 31st 2009, resulting in a follow-up period ranging from 1 to 60 months (median 49.0 months). The cases lost to follow-up and deaths from any cause other than gastric cancer were considered censored data during the analysis of survival rates.

In addition, we examined 154 endoscopic submucosal dissection (ESD) specimens for the treatment of early gastric cancer and dysplasia at Seoul National University College of Medicine between 2008 and 2010. Of these, 75 early gastric cancers consisted of 65 intestinal type and 10 diffuse type. All 79 cases of gastric epithelial dysplasia were adenomatous type, and consisted of 49 low-grade and 30 high-grade dysplasias. No foveolar type gastric dysplasias were included. A total of 90 non-neoplastic gastric mucosa specimens obtained from patients, who underwent surgical resection or ESD, were selected and they included 24 of normal mucosa, 30 cases of active gastritis, and 36 of intestinal metaplasia. The study protocol was reviewed and approved by the Institutional Review Board of Seoul National University Hospital.

Tissue array methods

Core tissue biopsies ( $2 \mathrm{~mm}$ in diameter) were taken from individual paraffin-embedded gastric tumors (donor blocks) and arranged in new recipient paraffin blocks (tissue array blocks) using a trephine apparatus 
(Superbiochips Laboratories, Seoul, Korea). A core was chosen from each case for analysis. We defined an adequate case as a tumor occupying more than $10 \%$ of the core area. Each tissue array block contained up to 60 cases, allowing 12 array blocks to contain a total of 653 cases.

\section{Immunohistochemistry}

Antibodies to $\mathrm{Lgl} 2$ and 7 EMT-related proteins were selected. After a test procedure using a human control slide (Superbiochips Laboratories), immunohistochemistry was performed with Lgl2 (1:500 dilution; Abnova, Walnut, CA, USA), E-cadherin (1:400 dilution; BD Biosciences, San Jose, CA, USA), $\beta$-catenin (1:800 dilution; BD Biosciences), MMP2 (1:200 dilution; Neomarkers, Fremont, CA, USA), S100A4 (1:500 dilution; DAKO, Carpinteria, CA, USA), Snaill (1:200 dilution; Abcam, Cambridge, MA, USA), zincfinger E-box-binding homeobox 1 (ZEB1) (1:100 dilution; Novus Biologicals, Littleton, CO, USA), and vimentin (1:1500 dilution; Neomarkers). After an antigen retrieval procedure using a microwave, primary antibodies were applied and antibody binding was detected using the avidin-biotin-peroxidase complex (Universal Elite ABC kit PK-6200; Vectastain, Burlingame, CA, USA) for $10 \mathrm{~min}$ and diaminobenzidine tetrahydrochloride solution (Kit HK153-5 K; Biogenex, San Ramon, CA, USA).

\section{Evaluation of immunohistochemistry}

The immunoreactivity of $\mathrm{Lgl} 2$ was graded in a semiquantitative manner $[17,19]$. The patterns of Lgl2 staining were classified into membrane (including basolateral pattern), cytoplasmic, and negative. The intensity of the staining was subjectively graded as negative, weak, moderate, or intense. Tumors were considered as loss of membranous expression when membrane staining in $<10 \%$ of the cells of interest. Additionally, when a mixed pattern was observed in a specimen without membranous expression, the major pattern (immunoreactivity $\geq 50 \%$ cells of interest) and the minor pattern (immunoreactivity $<50 \%$ cells of interest) were recorded as described previously [17-19].

E-cadherin and $\beta$-catenin were expressed in the membrane of non-neoplastic glands. Some of the gastric cancer cells showed loss or reduced expression of E-cadherin and nuclear expression of $\beta$-catenin. MMP2, S100A4, Snail1, vimentin, and ZEB1 were not expressed in non-neoplastic gastric epithelial cells. The altered expression of S100A4, Snaill, and ZEB1 was observed in the nucleus of tumor cells. MMP2 and vimentin were aberrantly expressed in the cytoplasm of tumor cells. For statistical analysis, the results of immunostaining were classified according to the proportion of positive tumor cells. When more than $75 \%$ of the tumor cells showed homogenous membranous E-cadherin staining, the tumor was considered positive. The expression of $\beta$-catenin, MMP2, S100A4, Snail1, vimentin, and ZEB1 was considered to be positive when $10 \%$ or more of the tumor cells were immunoreactive, as in previous study [23].

\section{RNA in situ hybridization}

In situ detection of $L g l 2$ mRNA was performed in 218 gastric tissue samples of ESD specimens by using the RNAscope kit (Advanced Cell Diagnostics, Hayward, CA, USA) according to the manufacturer's instructions. Briefly, $4 \mathrm{~mm}$ Formalin-fixed, paraffin-embedded (FFPE) tissue sections were pretreated by heating and protease application prior to hybridization with a target probe to Lgl2. A detailed procedure was described previously [24]. Positive staining was indicated by brown punctate dots present in the nucleus and/or cytoplasm. The expression was scored according to the instructions in the RNAscope FFPE assay kit: no staining (score 0), staining that was difficult to see under the $40 \times$ objective lens in more than $5 \%$ of tumor cells (score 1), staining that was difficult to see under the $20 \times$ objective lens but easy under the $40 \times$ objective lens in more than $5 \%$ of tumor cells (score 2), staining that was difficult to see under the $10 \times$ objective lens but easy under the $20 \times$ in more than $5 \%$ of tumor cells (score 3 ), and easy to see under the $10 \times$ objective lens in more than $5 \%$ of tumor cells (score 4). For statistical analysis, all cases were classified into 2 groups according to scores of mRNA level (score $0-1$ vs. 2-4). Sections stained for the housekeeping gene Ubiquitin $C(U b C)$ were also included as a positive control in each case. Samples with weak $U b C$ signals (score $\leq 2$ ) were excluded from analysis due to low RNA quality.

Statistical analysis

Associations between Lgl2 expression and clinicopathological factors were evaluated by $\chi^{2}$ test, Fisher's exact test, or Mann-Whitney $U$ test. Correlation coefficients between the expression of Lgl2 and EMT-related markers were estimated by the Spearman $\rho$ test. Survival curves were estimated using the Kaplan-Meier product-limit method, and the significances of differences between the survival curves were determined using the log-rank test. Multivariate survival analysis was performed using the Cox proportional hazards model. A $P$ value $<0.05$ was regarded as statistically significant. All statistical analyses were performed by using the SPSS version 19.0 (IBM SPSS Inc., Chicago, IL, USA). 


\section{Results}

Association between $\operatorname{Lgl} 2$ expression and clinicopathological factors in gastric cancer

The patterns of Lgl2 staining in conventional gastric cancers ranged from membranous and/or cytoplasmic staining to negative staining of all cancer cells (Fig. 1a-d). Tumor cells with membranous staining were usually accompanied with cytoplasmic staining. Overall, Lgl2 immunoreactivity was intact in $62(15.2 \%)$ cases of gastric cancer. Data representing the relationship between the pattern of $\mathrm{Lgl} 2$ staining and the clinicopathological factors of 409 gastric cancer samples are summarized in Table 1 . There were significant differences in Lgl2 staining patterns between the different histological types of adenocarcinoma according to WHO classification and Lauren's classification. Loss of membrane Lgl2 expression was significantly more frequent in diffuse type gastric cancers than in intestinal types $(P<0.001)$. Loss of membrane expression was correlated with large tumor size $(P=0.015)$, lymph node metastasis $(P=0.001)$ and advanced pTNM stage $(P=0.021)$, and a trend toward the depth of invasion $(P=0.088)$. No association was detected for age, sex, venous invasion, or distant metastasis.

In addition, gastric adenocarcinomas are histologically classified into two major types, intestinal and diffuse type $[22,25]$. These different types exhibit distinct biological behaviors and molecular signatures [22, 26-28]. Thus, we compared the clinicopathological features and the expression pattern of EMT-related proteins of both types of gastric cancer. We found that diffuse-type cancers were frequently observed in young female and significantly associated with advanced stage, lymph node metastasis, and distant metastasis as well as aberrant expression of E-cadherin and vimentin (Supplementary Table 1).
Fig. 1 Representative immunohistochemical features of gastric cancer. Membrane Lg12 expression with or without cytoplasmic staining in intestinal type adenocarcinoma (a, b). Diffuse cytoplasmic and negative staining in diffuse type adenocarcinoma (c, d). Gastric cancer cells showing nuclear S100A4 expression (e) and cytoplasmic MMP2 expression (f). (Original magnification $\times 400$; insets, higher magnification)
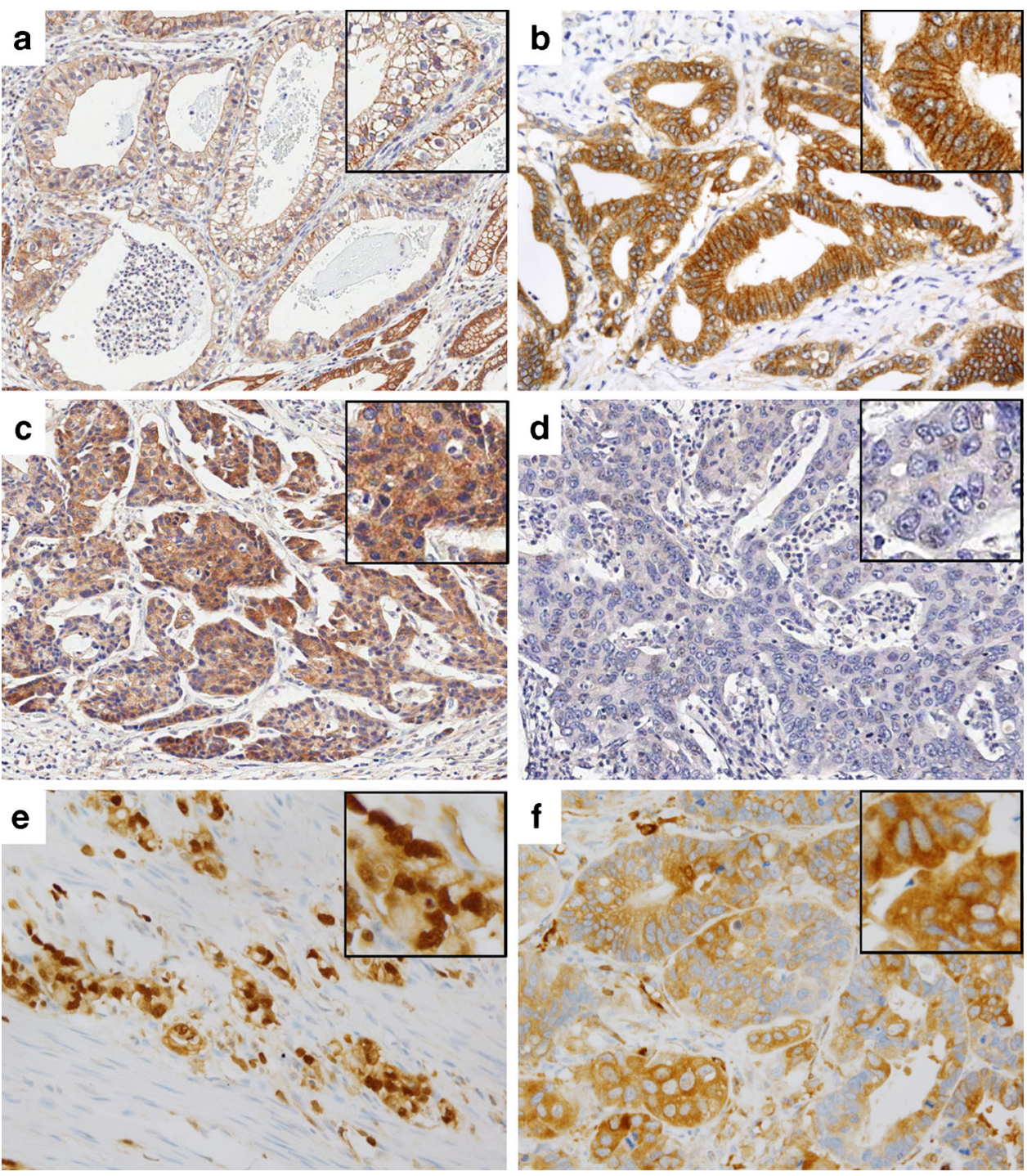
Table 1 Correlation between clinicopathological characteristics and Lgl2 expression in 409 gastric cancer (membrane expression vs. cytoplasmic/negative expression)
Characteristics

Membrane expression of $\mathrm{Lgl} 2$

$P$ value $^{\mathrm{a}}$

Presence $(n=62)$

Loss $(n=347)$

Age (years)

$\leq 65$

$>65$

$44(15.9)$

18 (13.6)

$233(84.1)$

$114(86.4)$

Gender

Male

50 (16.9)

$245(83.1)$

0.104

Female

$12(10.5)$

$102(89.5)$

Tumor size $(\mathrm{cm})$

Mean \pm SD

$4.74 \pm 2.39$

$5.77 \pm 3.15$

0.015

WHO classification

Papillary

$0(0)$

WD

14 (45.2)

MD

$40(27.0)$

$2(100)$

$<0.001$

PD

Mucinous

$5(3.8)$

$0(0)$

SRC

Undifferentiated

$3(4.1)$

$0(0)$

$0(0)$

Lauren's classification

Intestinal

54 (29.5)

8 (3.5)

Diffuse

Depth of invasion

EGC

$22(20.2)$

40 (13.3)

$\mathrm{T}$ stage

$\mathrm{T} 1$

$\mathrm{T} 2$

T3

$\mathrm{T} 4$

$22(20.2)$

11 (16.7)

19 (13.6)

$10(10.6)$

Lymph node metastasis

Absent

38 (22.4)

$24(10.0)$

Present

Distant metastasis

Absent

$58(16.3)$

(\%)

$S D$ standard deviation, $W D$

well-differentiated, $M D$

moderately differentiated, $P D$

poorly differentiated, $S R C$

signet ring cell carcinoma, $E G C$ early gastric cancer, $A G C$ advanced gastric cancer

a $\chi^{2}$ test, Fisher's exact test or Mann-Whitney $U$ test. A

$P$ values $<0.05$ was regarded as statistically significant
$4(7.5)$

31 (22.6)

12 (13.2)

15 (11.7)

$4(7.5)$

Present

pTNM stage

III

IV

Lymphatic invasion

Absent

31 (19.4)

$31(12.4)$

Present

$51(15.3)$

$11(14.7)$

36 (17.6)

26 (12.7)

Present

Perineural invasion
0.553

17 (54.8)

108 (73.0)

128 (96.2)

15 (100)

70 (95.9)

4 (100)

3 (100)

129 (70.5)

$<0.001$

$218(96.5)$

87 (79.8)

0.088

$260(86.7)$

87 (79.8)

0.259

55 (83.3)

$121(86.4)$

84 (89.4)

132 (77.6)

$<0.001$

$215(90.0)$

298 (83.7)

0.098

49 (92.5)

106 (77.4)

0.021

79 (86.8)

113 (88.3)

49 (92.5)

129 (80.6)

0.057

218 (87.6)

283 (84.7)

0.895

64 (85.3)

$\begin{array}{ll}\text { Absent } & 36(17.6) \\ \text { Present } & 26(12.7)\end{array}$

$169(82.4)$ 
Table 2 Correlation coefficients of expression status between the EMT markers in gastric cancer

\begin{tabular}{|c|c|c|c|c|c|c|c|c|}
\hline & Lg12 & $\beta$-catenin & E-cadherin & MMP2 & S100A4 & Snail1 & Vimentin & ZEB1 \\
\hline Lg12 & 1 & 0.010 & $0.220 * *$ & $0.105^{*}$ & -0.045 & -0.015 & $-0.131 * *$ & 0.027 \\
\hline$\beta$-catenin & & 1 & 0.010 & 0.009 & -0.015 & -0.042 & -0.054 & -0.039 \\
\hline E-cadherin & & & 1 & $0.229 * *$ & $-0.107 *$ & $-0.132 *$ & $-0.137 * *$ & -0.048 \\
\hline MMP2 & & & & 1 & 0.060 & -0.032 & -0.091 & 0.008 \\
\hline S100A4 & & & & & 1 & 0.060 & -0.005 & -0.089 \\
\hline Snail1 & & & & & & 1 & 0.086 & $0.114 *$ \\
\hline Vimentin & & & & & & & 1 & 0.055 \\
\hline ZEB1 & & & & & & & & 1 \\
\hline
\end{tabular}

* $P<0.05$

$* * P<0.01$

Correlation between Lgl2 expression and EMT-related proteins

To investigate the relationship between EMT-related proteins, expression levels were compared using the Spearman $\rho$ test (Table 2). Membrane Lgl2 expression was significantly correlated with E-cadherin expression $(\rho=0.220$, $P<0.001)$ and inversely correlated with vimentin expression $(\rho=-0.131, P=0.009)$. Loss of E-cadherin expression was significantly correlated with S100A4, Snail1, and vimentin $(P=0.044, \quad P=0.012$, and $P=0.09$, respectively). MMP2 expression was significantly correlated with $\mathrm{Lgl} 2$ and E-cadherin expression $(P=0.038$ and $P<0.001$, respectively). Snail1 and ZEB1 were significantly correlated with each other $(P=0.023)$.

Protein expression status and survival analysis

Overall patient survival rates were determined using the log rank test with respect to the expression of the eight proteins. Loss of membrane $\mathrm{Lgl} 2$ expression was found to be significantly associated with a poor outcome $(P=0.047)$ (Fig. 2a). Among EMT-related proteins, the expression of S100A4 $(P<0.001)$ (Fig. $2 b)$ and MMP2 $(P=0.021)$ (Fig. $2 \mathrm{c})$ was significantly associated with an unfavorable prognosis. However, no significant differences in patient outcome were found with respect to $\beta$-catenin, E-cadherin, Snail1, vimentin, or ZEB1 expression status (data not shown). To predict patient outcome more precisely, we performed a combined evaluation of EMT proteins after feature selection of three proteins including Lg12, S100A4, and MMP2, which showed a significant $P$ value in univariate survival analysis using the KaplanMeier method. For statistical analysis, cases were classified into two groups according to the number of proteins changed. Of the 409 cases enrolled, data on three proteins were available in 381 cases. $231(60.6 \%)$ cases had the aberrant expression of none or one protein and 150
(39.4\%) cases had an aberrant expression of two or three proteins. The patients who had tumors with a higher number of proteins changed had significantly worse prognosis than the other patients $(P<0.001)$ (Fig. $2 \mathrm{~d})$. Multivariate analysis revealed that a combined status of three proteins was an independent prognostic factor $(P=0.007)$ (Table 3).

\section{Lg12 expression status during gastric carcinogenesis}

The pattern of Lgl2 immunostaining in non-neoplastic gastric mucosa and neoplastic tissue samples was summarized in Figs. 3 and 4a. Lgl2 was expressed in a basolateral membrane pattern with absence of staining at the apical cell surface in all but one $(95.8 \%)$ case of normal mucosa (Fig. 3a). Most of the samples $(90.0 \%)$ with active gastritis showed a basolateral pattern of Lgl2 staining (Fig. 3b). Compared with normal mucosa, intestinal metaplasia showed various patterns of $\mathrm{Lgl} 2$ staining (Fig. 3c, d). Among 36 cases of intestinal metaplasia, membrane Lg12 expression was detected in 9 (25.0\%) cases (Fig. 4a). Cytoplasmic staining was the only or major pattern in $16(44.4 \%)$ cases and negative staining was the only or major pattern in $11(30.6 \%)$ cases.

Membrane Lg12 expression gradually decreased from gastric adenoma $(41.8 \%)$ and intestinal type $(30.6 \%)$ to diffuse type $(3.8 \%)$ gastric cancer (Fig. 4a). Of the 46 gastric adenomas with loss of membrane Lgl2 expression, 25 (14 low grade dysplasia and 11 high grade) showed cytoplasmic staining and 21 (15 low grade dysplasia and 6 high grade) showed absence of staining as the only or major pattern. No significant association was found between the $\mathrm{Lgl} 2$ staining pattern and the histological grade of dysplasia. In intestinal type gastric cancer, 125 $(50.4 \%)$ cases showed cytoplasmic staining and 47 $(19.0 \%)$ showed absence of staining as the only or major pattern. In diffuse type gastric cancer, cytoplasmic staining was observed in $105(44.5 \%)$ cases and negative staining 
Fig. 2 Survival analysis of 3 markers using the KaplanMeier method by the log rank test. Patients who have tumors with aberrant expression of 2 or 3 of these proteins have significantly poorer prognosis than the other patients. a $\mathrm{Lgl} 2$ $(P=0.047)$; b S100A4

$(P<0.001) ; \mathbf{c}$ MMP2

$(P=0.021)$; d combined evaluation of 3 markers $(P<0.001)$
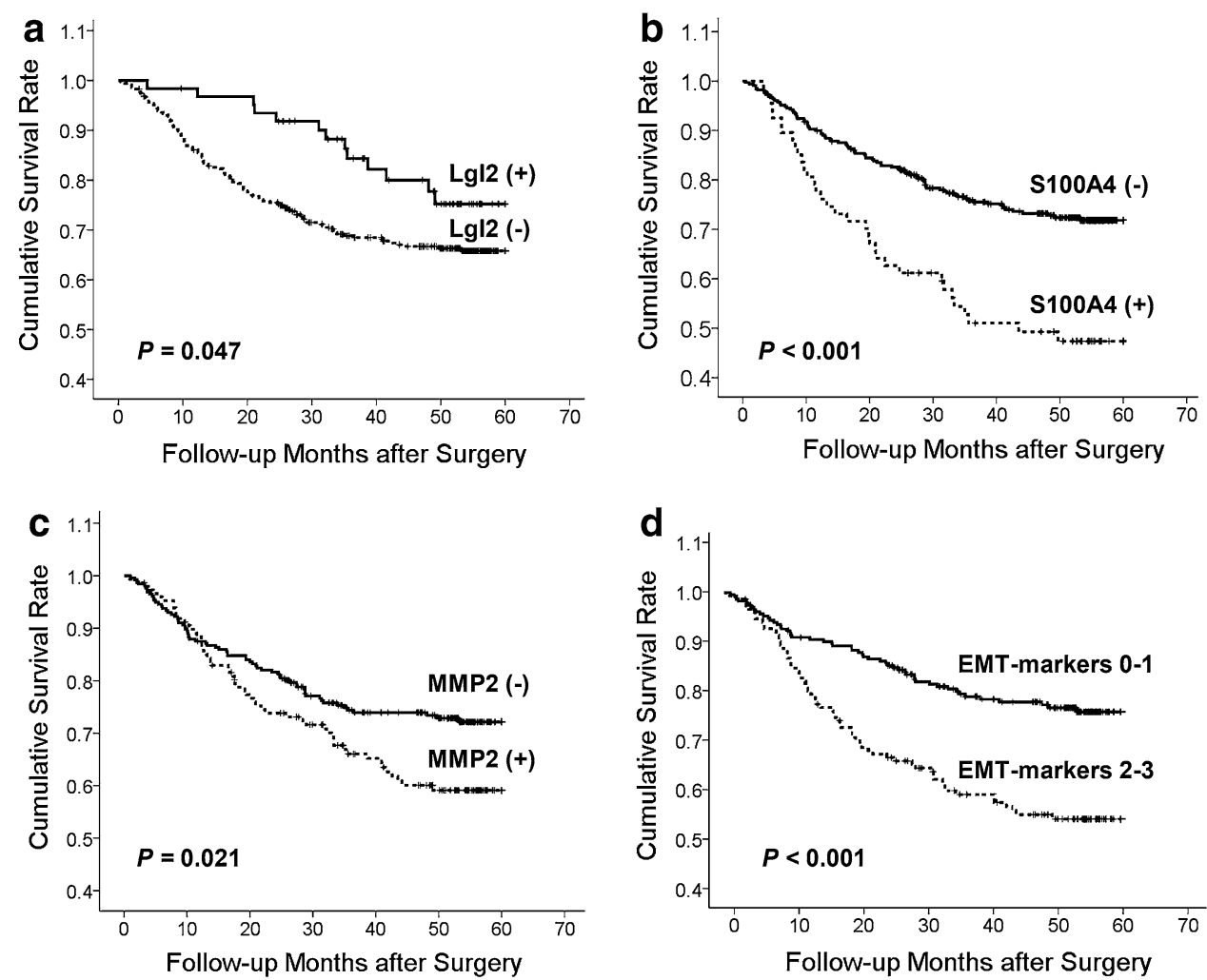

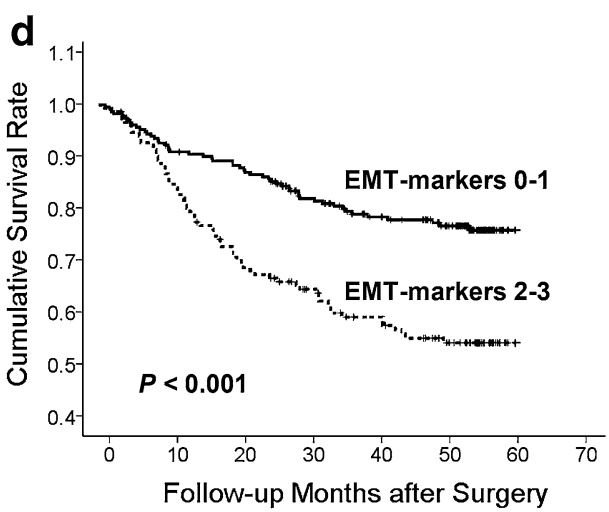

Table 3 Multivariate analysis of predictive factors for survival in gastric cancer patients (Cox proportional hazards model)

\begin{tabular}{llll}
\hline Variables & HR & $95 \%$ CI & $P$ value \\
\hline $\begin{array}{l}\text { Combination of 3 proteins } \\
0-1 \text { vs. 2-3 }\end{array}$ & 1.781 & $1.225-2.589$ & 0.002 \\
pTNM stage & & & \\
$\quad$ I-II vs. III-IV & 17.505 & $9.118-33.607$ & $<0.001$ \\
$\begin{array}{l}\text { Lauren's classification } \\
\quad \text { Intestinal type vs. diffuse type }\end{array}$ & 1.664 & $1.126-2.458$ & 0.011 \\
\hline
\end{tabular}

$H R$ hazard ratio, $C I$ confidence interval

was observed in $122(51.7 \%)$ cases as the only or major pattern.

Correlation between protein and mRNA expression of $\mathrm{Lgl} 2$

mRNA expression of $L g l 2$, as measured by mRNA in situ hybridization, correlated with protein expression, measured by immunohistochemistry (Fig. 4b). Of the 218 cases of gastric tissue samples, $12(11.6 \%)$ cases were scored 0,30 $(3.3 \%)$ cases scored 1, $64(13.5 \%)$ cases scored 2, 72 $(39.5 \%)$ cases scored 3, and $40(32.1 \%)$ cases scored 4 (Supplementary Fig. 1). Tumors with positive Lgl2 immunoreactivity showed significantly higher $L g l 2$ mRNA levels than those with negative immunoreactivity $(P<0.001)$. However, no significant difference in mRNA levels was found between tumors with membrane immunoreactivity and those with cytoplasmic immunoreactivity as the major pattern.

\section{Discussion}

Deregulated signaling of cell polarity complexes has been suggested to contribute to the multistep process of carcinogenesis $[8,29]$. Recently, it has been reported that loss of Lg12 results in the disruption of cell polarity and alteration of morphology, as well as promoting tumor progression in a variety of human epithelial cancers [4, 8, 14-17]. Despite several studies showing tumor suppressive activity of Lgl2 in various epithelial tumors, no reports have shown any clinical significance associated with Lg12 expression in gastric cancer. In the present study, we investigated its roles and relationship to clinicopathologic features in a large series of gastric cancer patients. This study is the first to document that loss of membrane $\mathrm{Lgl} 2$ expression was associated with aggressive behavior and poor prognosis in gastric cancer. Furthermore, deregulation of $\mathrm{Lgl} 2$ was associated with reduced expression of E-cadherin and the expression of vimentin, suggesting its involvement in EMT process. Taken together, we found that membrane Lgl2 
Fig. 3 Representative features of Lg12 immunoreactivity. Basolateral membrane expression of Lgl2 in normal gastric mucosa (a) and active gastritis (b). Cytoplasmic and negative staining in intestinal metaplasia (c, d). Membrane expression with cytoplasmic staining in gastric adenoma (e). Diffuse cytoplasmic staining in early gastric cancer $(\mathbf{f})$.

(Original magnifications $\times 200$; insets $\times 400$ )
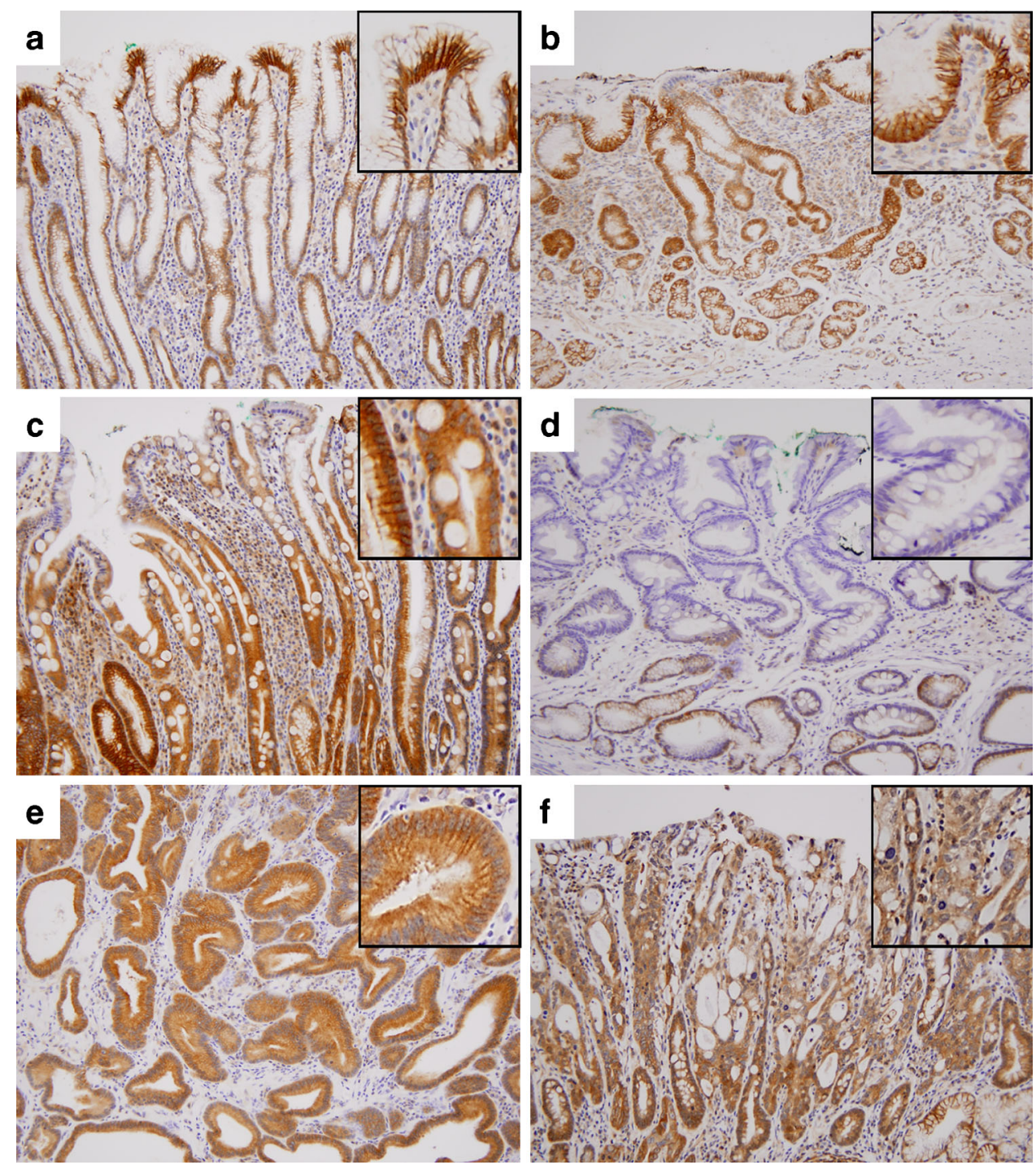
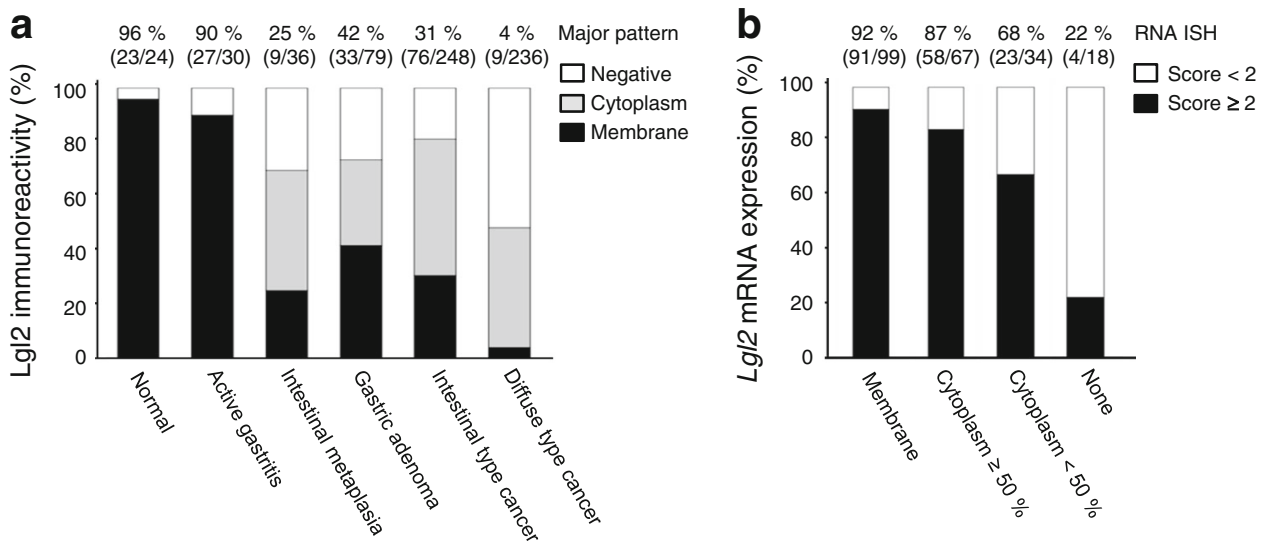

Fig. 4 a $\mathrm{Lgl} 2$ expression levels during gastric carcinogenesis. The frequency of membrane $\mathrm{Lgl} 2$ expression is gradually decreased in the normal-adenoma-carcinoma sequence. Percentages represent the number of samples with membrane expression per total sample population for a specific lesion. b Correlation between Lgl2 mRNA expression and protein abundance. A high correlation is found between RNA in situ hybridization and immunohistochemical results. Percentages represent the number of samples with scores $\geq 2$ of mRNA per total sample population for a specific pattern of immunoreactivity 
expression gradually decreased in normal-adenoma-carcinoma sequence. Therefore, we believe that our findings expand the body of evidence of the tumor suppressive role of Lgl2 associated with EMT in gastric cancer.

In the present study, we investigated the immunohistochemical characteristics of Lgl2 in a large series of gastric cancer tissue samples in order to clarify the clinicopathological significance of $\mathrm{Lgl} 2$ loss. We demonstrated that loss of membrane Lgl2 expression in gastric cancer was associated with aggressive tumor phenotype such as large tumor size, lymph node metastasis, advanced stage and poor prognosis. Although the mechanisms underlying how impaired cell polarity in absence of $\mathrm{Lgl} 2$, enables epithelial cells to gain malignant properties remains to be elucidated, our findings indicate that $\mathrm{Lgl} 2$ may act as a tumor suppressor in gastric cancer. This is consistent with published reports of various human cancers [14-17].

Studies on cell polarity proteins have shown that not only protein levels but also proper localization is essential for their functions including regulating cell growth and invasiveness [3, 5, 7]. Since alterations of transcript levels and protein abundance of Lgl2 have been reported in cancers [14-17, 30], we investigated whether changes in the protein expression levels of Lgl2 is associated with clinicopathological factors, compared to the effects of mislocalization. When we classified gastric cancers into 2 groups according to the protein expression levels of $\mathrm{Lgl} 2$ without regard to its localization, low expression (immunoreactivity $<50 \%$ of tumor cells) was not correlated with any of the clinicopathological factors of aggressive tumor behavior except for histological type (Supplementary Table 2). Collectively, although the molecular mechanisms of membrane localization of Lgl2 are largely unknown, our results suggest that alterations in proper membrane localization rather than protein abundance may be associated with a tumor suppressive role in gastric cancer. Furthermore, we investigated $L g l 2$ mRNA expression levels using mRNA in situ hybridization in gastric tissue specimens. Our results revealed a high degree of concordance between mRNA levels and protein abundance, but mRNA expression was correlated with both membrane and cytoplasmic expression of Lgl2 protein. Therefore, the results suggest that the amount of $L g l 2 \mathrm{mRNA}$ does not correlate with the tumor suppressor function of Lgl2.

Recently, a close relationship between the loss of cell polarity and the EMT process has been documented [8, 29, 31]. Based on previous data that showed that Lgl2 was a target of EMT transcription factors [14-16], we analyzed protein expression profiles in gastric cancer tissue samples to elucidate the potential connection between EMT-related proteins and Lg12. Our findings showed that Lgl2 expression was positively correlated with E-cadherin expression and inversely correlated with vimentin expression. By contrast, no significant correlation was detected between Lg12 and Snail1 or ZEB1, the key transcription factors of EMT. These findings support previous reports that regulation of cell polarity by EMT-related molecules involves a complex system and depends on the tumor context [31,32]. In addition to a direct link between EMT inducers and transcriptional down-regulation of several polarity genes, there appear to be indirect mechanisms that regulate cell polarity components at the transcriptional and post-transcriptional levels. For instance, Snail mediated-repression of PTEN or active function of microRNAs in EMT regulation could affect apical-basal cell polarity [31, 32]. Also, the activation of TGF- $\beta$ signaling during EMT participates in the regulation of cell polarity [33]. Interestingly, EMT in Lg12-mutant cells is facilitated by down-regulation of E-cadherin via activation of ErbB2 signaling rather than Snail-dependent repression [30].

Regarding the regulation of EMT by loss of E-cadherin, transcriptional repression by Snail or ZEB1 has been proposed as predominant mechanism in many carcinomas [31, 32]. In this study, we showed that E-cadherin expression inversely correlated with Snail1 expression $(\rho=-0.132$, $P=0.012$ ), whereas no significant correlation of E-cadherin and ZEB1 expression was observed $(\rho=$ $-0.048, P=0.366$ ). These findings are not strictly in accordance with previous reports, but several apparently discrepant results have been reported. Recent studies showed that down-regulation of E-cadherin did not correlate with the expression of known transcriptional repressors including Snail1 and ZEB1 in gastric, pancreatic, and breast cancer tissue [34-36]. SNAIL1 mRNA transcripts were correlated with down-regulation of E-cadherin in diffuse type gastric cancers [37], but this was not confirmed in primary gastric cancer using immunohistochemical study [38]. In addition, E-cadherin can be regulated by other mechanisms such as genetic mutation, promoter hypermethylation, post-translational modification as well as the influence of the tumor microenvironment during cancer progression [9, 39-41]. Furthermore, the discrepancy may partly be explained by the application of different methods such as reverse transcriptase-PCR, Western blot, and immunohistochemistry or the use of different samples such as cultured cancer cells or human cancer tissue [41]. Therefore, our results suggested that the transcriptional regulation of E-cadherin in gastric cancer is complex and the contribution of different repressors is dependent on the specific cellular or tumor context $[31,32]$.

In addition to the interaction between Lgl2 and EMTrelated proteins, we evaluated the impact of $\mathrm{Lgl} 2$ and EMT-related proteins on prognosis in gastric cancer. Results showed that aberrant expression of Lgl2, S100A4, and MMP2, was significantly associated with poor prognosis. The expression of MMP and S100A4 has been 
shown to be related to an invasive phenotype and poor prognosis in gastric cancer [42, 43], but the prognostic significance of Lgl2 was unclear. Based on published results that analysis of more prognostic markers may lead to a more reliable estimation of clinical outcomes [23, 34, 44], we performed a combined evaluation of $\mathrm{Lgl} 2$, S100A4, and MMP2 expression for prognosis. Our results suggest that a combined evaluation of Lgl2, S100A4 and MMP2 expression might provide a more precise estimation of prognosis than an evaluation of the three proteins alone for gastric cancer.

We found a progressive loss of membrane Lgl2 expression from gastric adenoma to intestinal type and diffuse type gastric cancer $(58,69$, and $96 \%$, respectively). Basolateral membrane expression of $\mathrm{Lgl} 2$ was not observed in $75 \%$ of intestinal metaplasia samples, but was preserved in most cases of normal mucosa and active gastritis (more than $90 \%$ ). In contrast to our results of the gradual and progressive loss of membrane $\mathrm{Lgl} 2$ expression, previous studies have reported that positive $\mathrm{Lgl} 2$ staining can be a useful diagnostic marker to rule out gastric dysplasia or adenocarcinoma [17]. Further validation studies are required to resolve this discrepancy.

In summary, we found that mislocalization of $\mathrm{Lgl} 2$ correlates with cancer progression and poor prognosis. Additionally, Lgl2 membrane expression is gradually decreased from premalignant lesion to carcinoma. These findings suggest that Lgl2 may function as a tumor suppressor in gastric cancer, depending on its proper localization. Our data show that membrane Lgl2 expression correlates with E-cadherin expression and a combined analysis of Lg12 with EMT markers, S100A4, and MMP2, could provide a more precise estimation of prognosis for patients with gastric cancer, which indicates a close relationship between Lg12 and EMT. Collectively, our results suggest that the loss of membrane Lgl2 expression occurs at an early stage of gastric carcinogenesis and contributes to gastric cancer progression related to EMT.

\section{References}

1. Jemal A, Bray F, Center MM, Ferlay J, Ward E, Forman D. Global cancer statistics. CA Cancer J Clin. 2011;61(2):69-90.

2. Vogiatzi P, Vindigni C, Roviello F, Renieri A, Giordano A. Deciphering the underlying genetic and epigenetic events leading to gastric carcinogenesis. J Cell Physiol. 2007;211(2):287-95.

3. Ellenbroek SI, Iden S, Collard JG. Cell polarity proteins and cancer. Semin Cancer Biol. 2012;22(3):208-15.

4. Martin-Belmonte F, Perez-Moreno M. Epithelial cell polarity, stem cells and cancer. Nat Rev Cancer. 2012;12(1):23-38.

5. Grifoni D, Garoia F, Bellosta P, Parisi F, De Biase D, Collina G, et al. aPKCzeta cortical loading is associated with Lgl cytoplasmic release and tumor growth in Drosophila and human epithelia. Oncogene. 2007;26(40):5960-5.
6. Bilder D, Li M, Perrimon N. Cooperative regulation of cell polarity and growth by Drosophila tumor suppressors. Science. 2000;289(5476):113-6.

7. Humbert PO, Grzeschik NA, Brumby AM, Galea R, Elsum I, Richardson HE. Control of tumourigenesis by the Scribble/Dlg/ Lgl polarity module. Oncogene. 2008;27(55):6888-907.

8. Royer C, Lu X. Epithelial cell polarity: a major gatekeeper against cancer? Cell Death Differ. 2011;18(9):1470-7.

9. Graziano F. Prognostic analysis of E-cadherin gene promoter hypermethylation in patients with surgically resected, node-positive, diffuse gastric cancer. Clin Cancer Res. 2004;10(8):2784-9.

10. Kuphal S, Wallner S, Schimanski CC, Bataille F, Hofer P, Strand $\mathrm{S}$, et al. Expression of Hugl-1 is strongly reduced in malignant melanoma. Oncogene. 2005.

11. Lu X, Feng X, Man X, Yang G, Tang L, Du D, et al. Aberrant splicing of Hugl-1 is associated with hepatocellular carcinoma progression. Clin Cancer Res. 2009;15(10):3287-96.

12. Schimanski CC, Schmitz G, Kashyap A, Bosserhoff AK, Bataille F, Schafer SC, et al. Reduced expression of Hugl-1, the human homologue of Drosophila tumour suppressor gene $\mathrm{lgl}$, contributes to progression of colorectal cancer. Oncogene. 2005;24(19):3100-9.

13. Tsuruga $T$, Nakagawa $S$, Watanabe M, Takizawa S, Matsumoto Y, Nagasaka K, et al. Loss of Hugl-1 expression associates with lymph node metastasis in endometrial cancer. Oncol Res. 2007;16(9):431-5.

14. Aigner K, Dampier B, Descovich L, Mikula M, Sultan A, Schreiber $\mathrm{M}$, et al. The transcription factor ZEB1 (deltaEF1) promotes tumour cell dedifferentiation by repressing master regulators of epithelial polarity. Oncogene. 2007;26(49):6979-88.

15. Kashyap A, Zimmerman T, Ergul N, Bosserhoff A, Hartman U, Alla $\mathrm{V}$, et al. The human Lgl polarity gene, $\mathrm{Hugl}-2$, induces MET and suppresses Snail tumorigenesis. Oncogene. 2013;32(11):1396-407.

16. Spaderna S, Schmalhofer O, Wahlbuhl M, Dimmler A, Bauer K, Sultan A, et al. The transcriptional repressor ZEB1 promotes metastasis and loss of cell polarity in cancer. Cancer Res. 2008;68(2):537-44.

17. Lisovsky M, Dresser K, Baker S, Fisher A, Woda B, Banner B, et al. Cell polarity protein Lg12 is lost or aberrantly localized in gastric dysplasia and adenocarcinoma: an immunohistochemical study. Mod Pathol. 2009;22(7):977-84.

18. Lisovsky M, Ogawa F, Dresser K, Woda B, Lauwers GY. Loss of cell polarity protein $\mathrm{Lgl} 2$ in foveolar-type gastric dysplasia: correlation with expression of the apical marker aPKC-zeta. Virchows Arch. 2010;457(6):635-42.

19. Patil DT, Bennett AE, Mahajan D, Bronner MP. Distinguishing Barrett gastric foveolar dysplasia from reactive cardiac mucosa in gastroesophageal reflux disease. Hum Pathol. 2013.

20. Edge SB, Byrd DR, Compton CC, Fritz AG, Greene FL, Trotti A. AJCC cancer staging manual. 7th edn. New York: Springer; 2010. pp. 117-26.

21. Bosman FT, Carneiro F, Hruban RH, Theise ND. WHO classification of tumours of the digestive system. 4th ed. Lyon: International Agency for Research on Cancer; 2010. pp. 46-80.

22. Lauren P. The two histological main types of gastric carcinoma: diffuse and so-called intestinal-type carcinoma. An attempt at a histo-clinical classification. Acta Pathol Microbiol Scand. 1965;64:31-49.

23. Kim MA, Lee HS, Lee HE, Kim JH, Yang HK, Kim WH. Prognostic importance of epithelial-mesenchymal transitionrelated protein expression in gastric carcinoma. Histopathology. 2009;54(4):442-51.

24. Ukpo OC, Flanagan JJ, Ma XJ, Luo Y, Thorstad WL, Lewis JS Jr. High-risk human papillomavirus E6/E7 mRNA detection by a novel in situ hybridization assay strongly correlates with p16 expression and patient outcomes in oropharyngeal squamous cell carcinoma. Am J Surg Pathol. 2011;35(9):1343-50. 
25. Japanese Gastric Cancer A. Japanese classification of gastric carcinoma-2nd English edition. Gastric Cancer. 1998;1(1):10-24.

26. Zheng H, Takahashi H, Murai $\mathrm{Y}$, Cui Z, Nomoto K, Miwa S, et al. Pathobiological characteristics of intestinal and diffuse-type gastric carcinoma in Japan: an immunostaining study on the tissue microarray. J Clin Pathol. 2007;60(3):273-7.

27. Sugai T, Habano W, Nakamura S, Yoshida T, Uesugi N, Suto T, et al. Correlation of histologic morphology and tumor stage with molecular genetic analysis using microdissection in gastric carcinomas. Diagn Mol Pathol. 1998;7(5):235-40.

28. Jinawath N, Furukawa Y, Hasegawa S, Li M, Tsunoda T, Satoh $\mathrm{S}$, et al. Comparison of gene-expression profiles between diffuseand intestinal-type gastric cancers using a genome-wide cDNA microarray. Oncogene. 2004;23(40):6830-44.

29. Etienne-Manneville S. Polarity proteins in migration and invasion. Oncogene. 2008;27(55):6970-80.

30. Reischauer S, Levesque MP, Nusslein-Volhard C, Sonawane M. Lg12 executes its function as a tumor suppressor by regulating ErbB signaling in the zebrafish epidermis. PLoS Genet. 2009;5(11):e1000720.

31. Moreno-Bueno G, Portillo F, Cano A. Transcriptional regulation of cell polarity in EMT and cancer. Oncogene. 2008;27(55):6958-69.

32. Thiery JP, Acloque H, Huang RY, Nieto MA. Epithelial-mesenchymal transitions in development and disease. Cell. 2009;139(5):871-90.

33. Lee M, Vasioukhin V. Cell polarity and cancer-cell and tissue polarity as a non-canonical tumor suppressor. J Cell Sci. 2008;121(8):1141-50.

34. Ryu HS, Park do J, Kim HH, Kim WH, Lee HS. Combination of epithelial-mesenchymal transition and cancer stem cell-like phenotypes has independent prognostic value in gastric cancer. Hum Pathol. 2012;43(4):520-8.

35. Montserrat N, Gallardo A, Escuin D, Catasus L, Prat J, GutierrezAvigno FJ, et al. Repression of E-cadherin by SNAIL, ZEB1, and TWIST in invasive ductal carcinomas of the breast: a cooperative effort? Hum Pathol. 2011;42(1):103-10.
36. Hotz B, Arndt M, Dullat S, Bhargava S, Buhr HJ, Hotz HG. Epithelial to mesenchymal transition: expression of the regulators snail, slug, and twist in pancreatic cancer. Clin Cancer Res. 2007;13(16):4769-76.

37. Rosivatz E, Becker I, Specht K, Fricke E, Luber B, Busch R, et al. Differential expression of the epithelial-mesenchymal transition regulators snail, SIP1, and twist in gastric cancer. Am J Pathol. 2002;161(5):1881-91.

38. Rosivatz E, Becker KF, Kremmer E, Schott C, Blechschmidt K, Hofler $\mathrm{H}$, et al. Expression and nuclear localization of Snail, an E-cadherin repressor, in adenocarcinomas of the upper gastrointestinal tract. Virchows Arch. 2006;448(3):277-87.

39. Becker KF, Kremmer E, Eulitz M, Becker I, Handschuh G, Schuhmacher C, et al. Analysis of E-cadherin in diffuse-type gastric cancer using a mutation-specific monoclonal antibody. Am J Pathol. 1999;155(6):1803-9.

40. Pinho SS, Figueiredo J, Cabral J, Carvalho S, Dourado J, Magalhaes A, et al. E-cadherin and adherens-junctions stability in gastric carcinoma: functional implications of glycosyltransferases involving $\mathrm{N}$-glycan branching biosynthesis, $\mathrm{N}$-acetylglucosaminyltransferases III and V. Biochim Biophys Acta. 2013;1830(3):2690-700.

41. Peinado H, Olmeda D, Cano A. Snail, Zeb and bHLH factors in tumour progression: an alliance against the epithelial phenotype? Nat Rev Cancer. 2007;7(6):415-28.

42. Alakus H, Grass G, Hennecken JK, Bollschweiler E, Schulte C, Drebber U, et al. Clinicopathological significance of MMP-2 and its specific inhibitor TIMP-2 in gastric cancer. Histol Histopathol. 2008;23(8):917-23.

43. Wang YY, Ye ZY, Zhao ZS, Tao HQ, Chu YQ. High-level expression of S100A4 correlates with lymph node metastasis and poor prognosis in patients with gastric cancer. Ann Surg Oncol. 2010;17(1):89-97.

44. Lee HS, Lee HK, Kim HS, Yang HK, Kim WH. Tumour suppressor gene expression correlates with gastric cancer prognosis. J Pathol. 2003;200(1):39-46. 